\title{
TRANSLATION PLANES OF EVEN ORDER IN WHICH THE DIMENSION HAS ONLY ONE ODD FACTOR
}

\author{
T. G. OSTROM \\ Department of Pure and Applied Mathematics \\ Washington State University \\ Pullman, Washington 99164 U.S.A.
}

(Received January 2, 1980)

ABSTRACT. Let $G$ be an irreducible subgroup of the linear translation complement of a finite translation plane of order $q^{d}$ where $q$ is a power of $2 . G F(q)$ is in the kernel and $d=2^{s} r$ where $r$ is an odd prime. A prime factor of $|G|$ must divide $\left(q^{d}+1\right) \underset{i=1}{d}\left(q^{i}-1\right)$.

One possibility (there are no known examples) is that $G$ has a normal subgroup $W$ which is a w-group for some prime w.

The maximal normal subgroup $O(G)$ satisfies one of the following:

1. Cyclic. 2. Normal cyclic subgroup of index $r$ and the nonfixed-point-free elements in $O(G)$ have order r. 3. $O(G)$ contains a group $W$ as above.

KEY WORDS AND PHRASES. Translation planes, collineation groups, finite geometries. 1980 MATHEMATICS SUBJECT CLASSIFICATION CODES. 50D35, 05B25, $20 B 25$. 
If $\mathrm{r}=3$ and $\mathrm{W}$ exists then $\mathrm{w}=3$ or $\mathrm{W}$ is elementary abelian and of order $W^{2}$. If $r=3, s=2$ and if $G$ is nonsolvable and contains no áfine elations, let $\mathrm{S}$ be the subgroup generated by the 2-elements. Then $\mathrm{S}=\mathrm{SL}\left(2,2^{\mathrm{b}}\right)$ or $\mathrm{S} / 0(\mathrm{~S})=\operatorname{PSL}(2, \mathrm{u})$ for $\mathrm{u}=5,11,13$, or 19 and $O(S)$ is trivial unless $u=5$.

We include some corrections to our paper "Translation planes of odd order and odd dimension".

\section{INTRODUCTION.}

For definitions of "spread", "translation complement", and other special terminology with respect to translation planes, see [9]. The reader is reminded that if the spread is defined on a vector space of dimension $2 \mathrm{~d}$, so that the components of the spread have dimension $d$ then it is customary to say that the translation plane has dimension $d$ (over its kernel). The class of known finite translation planes is very large but they tend to fall into a few classes and the members of each class are very much like each other. The class of linear groups which are known to act as subgroups of the translation complement is very limited. For translation planes of order $\mathrm{p}^{\mathrm{r}}$ ( $\mathrm{p}$ is prime) many have subgroups of small index which are subgroups of $\Gamma L\left(2, \mathrm{p}^{\mathrm{r}}\right)$ (the translation complement for Desarguesian planes). In a few cases, they are direct products of such groups. This is the case in the irregular nearfield planes and some related to them. In the semifield planes of order $\mathrm{p}^{\mathrm{r}}$, there are normal elementary abelian subgroups of order $p^{r}$ (affine elations - or shears). The Suzuki groups come in when $p=2$ and $d=2$. The only other cases which come to the author's mind are $\operatorname{SL}(2,7)$ and $\operatorname{SL}(2,13)$. The question arises: Which groups can act as subgroups of linear translation complements of translation planes? It may be unrealistic to expect a complete answer to this question. Nevertheless either there must be some more translation planes around that are quite different from the known ones and that 
admit different kinds of groups or there must be undiscovered restrictions on translation complements.

Let $G$ be a subgroup of the linear translation complement of a finite translation plane. A reasonable strategy would seem to be to identify certain key normal subgroups (and how they act on the plane): the minimal normal nonsolvable subgroups if $G$ is nonsolvable, the group generated by 2-elements if $|G|$ is even. Group theoretic arguments often give us factor groups of these subgroups with respect to normal subgroups of $G$ which are maximal in some sense. To understand the action of the minimal normal nonsolvable subgroup or the subgroup generated by the 2-elements, we must determine what the subgroups at this second stage are like. For instance, we may need to know something about the maximal normal subgroup of odd order.

In general, what happens is one of the following: (1) The "Second-leve1" normal subgroup is cyclic (2) $G$ is imprimitive and has a normal noncyclic elementary abelian group (3) G has a normal extra-special group.

Theorems that certain classes of groups cannot act on any finite translation plane are hard to come by. In practice we shall have to reword our question to read: For a given class of values of $q$ and of $d$, which groups can act on a translation plane of dimension $d$ over GF(q)? (For instance, we can go quite a long way towards answering this question when $q$ and $d$ are both odd.) What we end up with is a set of theorems which screen out certain groups. We should like then to have results which say that the survivors, if they do act on certain planes, must act in specified ways which are well enough defined for us to specify explicit representations which may enable us to construct the planes or, perhaps, to show that a plane admitting a particular group acting in a particular way cannot, in fact, exist.

In this paper we investigate the case where $q$ is a power of $2, G$ is 
irreducible, and $d=2^{\mathrm{S}} \mathrm{r}$ for some odd prime $\mathrm{r}$.

We do not claim to have any spectacular results. We believe that we use methods which can be developed and used to investigate other cases. We do have results as to how the groups act on the (possible) planes. We need more knowledge of representations to carry out the last phase of the program. Indeed the representation theory we need is probably quite different from that included in standard representation theory.

Section II deals with the nonsolvable case without restriction on the dimension. Much of it is just a summary of previous results except that the connection with extra-special groups is clarified.

In Section III we do restrict the dimension (and characteristic of the field) as mentioned above. Our main result in this section is Theorem (3.7) which gives the possibilities for the maximal normal subgroup of odd order $O(G)$ in terms of (1), (2), and (3) above.

In Section IV we look at planes still more explicitly, especially for the case $r=3$. With respect to the general question as to which subgroups of GL $(2 d, p)$ can be subgroups of the translation complement for a translation plane of order $\mathrm{p}^{\mathrm{d}}$, Theorem (4.8) says that certain primes cannot divide the order of the translation complement even if they divide $\mid G L(2 d), p) \mid$. We also make use of some results of Hering and Ho [5] for translation planes of dimension $2 \mathrm{r}$ over GF (q), where $r$ is odd and $q$ is even. Let $S$ be the subgroup of the linear translation complement which is generated by the 2-elements. Hering and Ho determine the nature of $\mathrm{S} / 0(\mathrm{~S})$. With $\mathrm{r}$ restricted to be prime we can say more about the possible nature of $O(S)$ and how $S$ acts on the plane if a plane does exist admitting a group $S$ in Hering and Ho's list. We have not been able to construct new planes furnishing examples but we can narrow down the possibilities. Some, but not all, of the other papers in the same spirit and the cases with 
which they deal are: odd order, odd dimension [12]; dimension 2 and characteristic 2 with all involutions Baer [7]; odd order, dimension 2 [11]; kernel GF(q) admitting $\operatorname{SL}(2, q)[15],[14],[8]$. We hope that these results are explicit enough to suggest to someone either how other types of planes may be constructed or how to obtain stronger nonexistence theorems.

This research was supported in part by the National Science Foundation.

The rest of this Introduction consists of preliminary definitions and results, as well as some matters of notation.

(1.1) DEFINITION. Let $G$ be a group of nonsingular linear transformation acting on a vector space $V$. Then $G$ is said to be fixed-point-free (f.p.f.) if no nontrivial member of $G$ fixes any nonzero vector.

(1.2) DEFINITION. Let $G$ be a group of nonsingular linear transformations acting on a vector space $V$. Then a normal subgroup $G_{0}$ is said to be minimal non-f.p.f. with respect to $G$ if $G_{0}$ is not f.p.f. but every normal subgroup of $G$ properly contained in $G_{0}$ is f.p.f.

(1.3) DEFINITION. With $G, V$ as (1.2) a subspace $V_{0}$ of $V$ is called a minimal G-space if $G$ leaves $V_{0}$ invariant and acts irreducibly on it. $A$ subspace $V_{1}$ is a homogeneous G-space if $V_{1}$ is invariant under $G$ and all of the minimal G-spaces in $V_{1}$ are isomorphic as G-modules. (When there is no doubt as to which group is being referred to, the $G$ may be omitted.)

REMARK - An f.p.f. group is a Frobenius complement. It is well known that the Sylow subgroups of odd order in a Frobenius complement are cyclic and the Sylow 2-groups are either cyclic or generalized quaternion.

Notation:

We shall be looking at vector spaces over the field GF(q) where $q$ is a power of the prime p. 
The order of the group $G$ is denoted by $|G|$.

We use $O(G)$ to denote the maximal normal subgroup of odd order.

If $H$ is a subgroup of $G_{1}, C(H)$ is the centralizer of $H$ in the full group $G$. If $G_{1}$ is a subgroup, the centralizer of $H$ in $G_{1}$ will be denoted by $C(H) \cap G_{1}$.

If $\sigma$ is a linear transformation acting on a vector space $\mathrm{V}, \mathrm{V}(\sigma)$ denotes the subspace consisting of all fixed points of $\sigma$.

Fit $G$ is the Fitting subgroup of $G$.

\section{THE NONSOLVABLE CASE.}

(2.1) LEMMA. Let $G$ be a nonsolvable group of linear transformations acting on a finite dimensional vector space $V$ over a finite field $F=G F(q)$ and let $G_{0}$ be a minimal nonsolvable normal subgroup. Then every normal cyclic subgroup of $G$ included in $G_{0}$ is in $Z\left(G_{0}\right)$. The Fitting subgroup of $G_{0}$ is equal to $Z\left(G_{0}\right)$ if it is fixed point free.

PROOF. See Lemmas $(2.1)$ and (2.2) in [12].

(2.2) LEMMA. In the notation of the previous Lemma, let $H$ be a norma1 subgroup of $G$ included in $G_{0}$ but not equal to $G_{0}$ and let $H$ be maximal with respect to this property. Then $G_{0} / H$ is a direct product of isomorphic simple groups.

If Fit $G_{0}$ is fixed point free then $H=$ Fit $G_{0}=Z\left(G_{0}\right)$.

PROOF. See the proof of Theorem (2.3) in [12].

(2.3) LEMMA. In the notation of the previous Lemmas, suppose that Fit $G_{0}$ is not fixed point free. Then $G_{0}$ includes a subgroup $W$ which is minimal non-f.p.f. group with respect to $G$, where $W$ is a w-group for some prime $w$. If $W_{0}$ is a maximal normal subgroup of $G$ included in $W$ but not equal to $W$, then $\mathrm{W}_{0} \subseteq \mathrm{Z}\left(\mathrm{G}_{0}\right)$ and $\mathrm{W} / \mathrm{W}_{0}$ is elementary abelian. 
PROOF. See Lemma (2.8) in [12].

(2.4) LEMMA. Suppose that $W$ of the previous Lemma is nonabelian. Then $W_{0}$ is cyclic and equal to $Z(W)$.

PROOF. Note that $\mathrm{W}_{0}$ is a fixed-point-free $\mathrm{w}$-group. Thus if $\mathrm{w}$ is odd, $\mathrm{W}_{0}$ must be cyclic. We already have that $\mathrm{W}_{0} \subseteq \mathrm{Z}\left(\mathrm{G}_{0}\right)$ by (2.1) so $\mathrm{W}_{0} \subseteq \mathrm{Z}(\mathrm{W})$. If $W$ is nonabelian $Z(W) \subseteq W$ must be included in $W_{0}$ so $W_{0}=Z(W)$ if $w$ is odd. If $w$ is even, then $W_{0}$ is either cyclic or generalized quaternion. If $\mathrm{W}_{0}$ is cyclic we can use the same argument that we used for odd $\mathrm{w}$. If $\mathrm{W}_{0}$ is generalized quaternion, then $\mathrm{W}_{0} \nsubseteq \mathrm{Z}\left(\mathrm{G}_{0}\right)$ and $\mathrm{G}_{0}$ must induce a nonsolvable group of automorphisms on $\mathrm{W}_{0}$ contrary to the fact that the automorphism group of a generalized quaternion group is nonsolvable.

(2.5) LEMMA. If $\mathrm{W}$ is nonabelian then $\mathrm{W}$ is extra-special. Furthermore:

(a) $\overline{\mathrm{W}}=\mathrm{W} / \mathrm{W}_{0}$ has square order; $\mathrm{W}_{0}$ has order $\mathrm{w}$.

(b) $\bar{W}$ is a symplectic space; if $|W|=w^{2 m+1}$

the automorphism group induced on $\bar{W}$ by $G$ is a subgroup of $\operatorname{Sp}(2 \mathrm{~m}, \mathrm{w})$.

(c) $\mathrm{W}$ is a central product of subgroups $\mathrm{w}_{1}, \mathrm{w}_{2}, \ldots, \mathrm{w}_{\mathrm{m}}$, where $\left|w_{i}\right|=w^{3}$ and $\bar{W}_{i}$ is elementary abelian of order $w^{2}$.

PROOF. This is essentially Satz 13.7 in Huppert [6].

(2.6) LEMMA. Suppose that $\mathrm{W}$ is nonabelian and that $|\mathrm{W}|=\mathrm{w}^{2 \mathrm{~m}+1}$. Then $\mathrm{w}^{\mathrm{m}}$ divides the dimension of the vector space if $G$ is irreducible.

PROOF. Let $W_{1}$ be a subgroup of order $w^{3}$, where $\bar{W}_{1}$ is elementary abelian of order $w^{2}$. Let $W_{0}=\langle\nu\rangle$. Let $\bar{W}_{1}=\langle\bar{\rho}, \sigma\rangle$, let $\rho, \sigma$ be preimages of $\bar{\rho}, \bar{\sigma}$ respectively so that $W_{1}=\langle\rho, \sigma, \nu\rangle$. Without loss of generality, $\mathrm{V}(\sigma)$ is nontrivial. Since $\mathrm{W}_{1}$ is nonabelian but $\overline{\mathrm{W}}_{1}$ is elementary abelian we can, without loss of generality, assume $\rho^{-1} \sigma \rho=\sigma \nu$. Then $\sigma \nu^{i}$ fixes $V(\sigma) \rho^{i}$ pointwise and

$$
\mathrm{V}(\sigma)+\mathrm{V}(\sigma) \rho+\ldots+\mathrm{V}(\sigma) \rho^{\mathrm{w}-1}=\mathrm{V}(\sigma) \oplus \mathrm{V}(\sigma) \rho \oplus \ldots \oplus \mathrm{V}(\sigma) \rho^{\mathrm{w}-1}
$$


Thus we have a vector space of dimension $w \operatorname{dim} V(\sigma)$ which is invariant under $\mathrm{W}_{1}$ - Call this vector space $\mathrm{V}_{1}$. Now $\mathrm{W}_{2}$ centralizes $\mathrm{W}_{1}$ and hence leaves each of $V(\sigma), V(\sigma) \rho$, etc. invariant. In fact, each of $V(\sigma)$, etc. contains a subspace of dimension divisible by $w$ which is invariant under $w_{1}$. Thus $\mathrm{W}_{1} \mathrm{~W}_{2}$ has invariant subspace whose dimension is a multiple of $w^{2}$.

By induction, $\mathrm{W}=\mathrm{W}_{1} \mathrm{~W}_{2} \ldots \mathrm{W}_{\mathrm{m}}$ has invariant subspace whose dimension is a multiple of $w^{m}$. If $G$ is irreducible on $V$, then $w^{m}$ divides the dimension $V$. REMARK. In [12] we obtained results similar to (2.5) and (2.6) by a more complicated argument.

In Lemmas $(2.1)-(2.6)$ we assumed that $G$ was nonsolvable. The next Lemma requires no assumption as to whether or not $G$ is solvable.

(2.7) LEMMA. Let $G$ be an irreducible group of linear transformations acting on a vector space $\mathrm{V}$ of dimension $\mathrm{n}$ over the field $\mathrm{F}$. Let $\mathscr{U}$ be a cyclic normal subgroup of $G$. Then the index of $C(2)$ in $G$ divides $n$ if $C(2)$ is faithful on the homogeneous $\mathscr{U}$-spaces.

PROOF. By Clifford's Theorem, we can write $V$ as a direct sum $\mathrm{V}=\mathrm{v}_{1} \oplus \ldots \oplus \mathrm{v}_{\mathrm{k}}$ of homogeneous 2 -spaces, where $\mathrm{n}=\mathrm{k} \operatorname{dim} \mathrm{v}_{1}$ and the stabilizer of $V_{1}$ has index $k$ in $G$ and $C(2)$ leaves $V_{1}$ invariant. By Hering [4] the index of $C(\mathscr{U})$ in the stabilizer of $v_{1}$ divides dim $v_{1}$. (2.8) LEMMA. Suppose that $G$ is irreducible and has a normal abelian non-f.p.f. w-group $W$, where $w$ is prime. Then $G$ is imprimitive with subspaces of imprimitivity $v_{1}, v_{2}, \ldots$ such that each $v_{i}$ is a homogeneous $w$-space and is pointwise fixed by some nontrivial subgroup $W\left(V_{i}\right)$ of $W$.

PROOF. Each homogeneous W-space is a direct sum of minimal W-spaces that are isomorphic as $\mathrm{W}$-modules. If $\mathrm{W}$ is elementary abelian (not cyclic) then $\mathrm{W}$ is not f.p.f. on any of its invariant subspace. If $\sigma \in W$ and $V(\sigma) \cap v_{1}$ is 
nontrivial then $\mathrm{V}(\sigma)$ is invariant under $\mathrm{W}$ and includes a minimal $\mathrm{W}$-space. Thus $\sigma$ fixes pointwise all minimal $\mathrm{W}$-spaces in $\mathrm{V}_{1}$ and hence fixes $\mathrm{V}_{1}$ pointwise. Here we would like to make certain corrections to [12]. In the proof of (2.9) in [12], we denoted $G / C(W)$ by $\hat{G}$, where $W$ was a non-f.p.f. w-group normal in $G$ and $W_{0}$ was the maximal fixed point free normal subgroup of $G$ included in $W$. We wished to show that, under the hypotheses, $W_{0}$ had to be trivial. We disposed of the case where the subgroup of $\hat{G}_{0}$ which centralizes $\hat{W}$ was nonsolvable but did not include the argument where this group might be solvable.

Let $\hat{H}_{1}$ be the subgroup of $\hat{G}_{0}$ which centralizes $\hat{W}$. If $\hat{H}_{1}$ is solvable, then $\hat{\mathrm{G}}_{0} / \hat{\mathrm{H}}_{1}$ must be nonsolvable since $\hat{\mathrm{G}}_{0}$ must be nonsolvable. 'Then $\hat{\mathrm{G}}_{0} / \hat{\mathrm{H}}_{1}$ is isomorphic to a nonsolvable subgroup of the automorphism group of $\hat{W}$ and hence to a nonsolvable subgroup of $\mathrm{GL}(2, w)$. By hypotheses, the Sylow 2-groups of $G / Z(G)$ are dihedral and this implies that the sylow 2-groups of $\hat{G}_{0} / \hat{\mathrm{H}}_{1}$ are cyclic or dihedral. But the nonsolvable subgroups of $\mathrm{GL}(2, \mathrm{w})$ do not have cyclic or dihedral sylow 2-groups. This disposes of the case where $\hat{H}_{1}$ is solvable.

LEMMA (3.3) and Theorem (3.4) of [12] should be deleted - the proofs do not appear to be correct. The statement of (3.5) should be altered to read:

THEOREM. Let $\Pi$ be a translation plane of order $q^{d}$ with kernel GF(q), where $\mathrm{q}$ and $\mathrm{d}$ are odd. Let $\mathrm{G}$ be a subgroup of the linear translation complement. Suppose that $\mathrm{G}$ is irreducible and is faithful on its subspaces of imprimitivity or is primitive. Suppose also that $d$ is the product of distinct primes. Let $G_{0}$ be a minimal nonsolvable normal subgroup of $G$. Then either $G_{0}=S L(2, u)$ for some odd $u$ or $\bar{G}_{0}=A_{6}$ or $A_{7}$. Here $\bar{G}_{0}=G_{0} / Z\left(G_{0}\right)$. 


\section{GROUPS OF ODD ORDER; DIMENSION HAS ONLY ONE ODD PRIME FACTOR.}

In this section $G$ is again assumed to be acting irreducibly on a vector space $\mathrm{V}$ of dimension $\mathrm{n}$ over $\mathrm{GF}(\mathrm{q})=\mathrm{F}$.

(3.1) Definition. The dimension will be assumed to be a power of 2 times an odd number. Specifically the dimension $\mathrm{n}$ is equal to $2^{\mathrm{s}} \mathrm{r}$ where $\mathrm{s}$ is an integer and $r$ is an odd prime or is equal to 1 . Also $F=G F(q)$ where $q=2^{t}, t \geqq 1$.

(3.2) LEMMA. If $|G|$ is odd and 2 is a maximal cyclic normal subgroup of $G$, then $\mathbb{C}(\mathfrak{U})$ has index in $G$ which divides $r$ provided $C(\mathscr{U})$ is faithful on the homogeneous $\mathscr{U}$-spaces.

PROOF. Use Clifford's theorem to write $V$ as a direct sum of homogeneous $\mathscr{U}$-spaces. The number of homogeneous $\mathscr{U}$-spaces must divide both $\mathrm{n}$ and $|G|$ and hence must divide $r$. As in the proof of (2.7), the index of $C(G)$ in the stabilizer of a homogeneous $\mathscr{U}$-space must also divide $(n,|G|)$. The remainder of the proof is also similar to the proof of (2.7).

(3.3) LEMMA. Suppose that $|G|$ is odd and that $G$ is irreducible. Then $G$ is cyclic if $G$ is f.p.f. If Fit $G$ is f.p.f. but $G$ is not, then the nonf.p.f. elements in $G$ have order $r$ and Fit $G$ is a cyclic normal subgroup of index $r$.

PROOF. Let $\mathrm{H}=\mathrm{Fit} \mathrm{G}$. Then $\mathrm{H}$ is fixed point free and thus is a Frobenius complement. The Sylow subgroups of odd order in a Frobenius complement are cyclic and $H$ is the direct product of its Sylow subgroups so $H$ is cyclic. Let $\mathrm{H}_{1}=\mathrm{C}(\mathrm{H})$. Then Fit $\mathrm{H}_{1}=\mathrm{H}$. The Fitting subgroup of a solvable nonabelian group is strictly greater than its center. This would imply that $H$ is strictly greater than $\mathrm{Z}\left(\mathrm{H}_{1}\right)$ if $\mathrm{H}$ were not cyclic. This would be a contradiction so $\mathrm{H}_{1}$ is cyclic and $\mathrm{H}_{1}=\mathrm{H}$. Hence (3.2) implies that $\mathrm{H}$ has index in $G$ which divides $r$. 
Thus if $G$ is non-f.p.f., the non-f.p.f. elements in $G$ must have order r. If $G$ is f.p.f. and $|G|$ is odd, then $G$ is a Z-group-i.e., all of its Sylow subgroups are cyclic.

Thus, $G$ is generated by two elements $\rho$ and $\sigma$ of relatively prime order, $\langle\sigma\rangle$ is normal in $G$ and $|G|=|\sigma||\rho|$. See Passman [13], p. 106. Let $\tau$ be an element of order $\mathrm{r}$. If $\langle\tau\rangle \subseteq\langle\sigma\rangle$ then $\langle\tau\rangle \triangleleft \mathrm{G}$ and $\mathrm{G}=\langle\tau, \mathrm{H}\rangle$ so $\mathrm{G}$ is cyclic. Otherwise $\langle\tau\rangle \subseteq\langle\rho\rangle$. By the argument on the top of p. 197 in Passman $\langle\tau\rangle$ must centralize $\langle\sigma\rangle$ in this case. But $\langle\tau\rangle$ must also centralize $\rho$ if $\langle\tau\rangle \subseteq\langle\rho\rangle$. We conclude that $G$ is cyclic if $G$ is f.p.f. (3.4) LEMMA. If $|G|$ is odd, if $G$ is irreducible and Fit $G$ is not f.p.f., then $G$ has a minimal non-f.p.f. group $W$, where $W$ is a $w$-group for some prime w. Either (a) $|W|=r^{3}, w=r, W / Z(W)$ is elementary abelian of order $r^{2}$ or (b) $\mathrm{W}$ is elementary abelian and $\mathrm{G}$ is imprimitive with $\mathbf{r}$ homogeneous W-spaces as subspaces of imprimitivity..

PROOF. Let $W_{0}$ be the maximal f.p.f. normal subgroup of $G$ included in $\mathrm{W}$ if $\mathrm{W}$ is nonabelian. Note that $\mathrm{w}$ must be odd if $\mathrm{q}$ is a power of 2 and $G$ is irreducible. If $C\left(W_{0}\right)$ is faithful on the homogeneous $W_{0}$ spaces, it follows from (2.7) that the index of $\mathcal{C}\left(W_{0}\right)$ in $G$ divides $2^{S} r$ so the index of $C\left(W_{0}\right) \cap W$ in $W$ must divide $r$. If $|W|=w^{m+1}$ then $\left|C\left(W_{0}\right)\right| \geqq w^{m}$. Then $C\left(W_{0}\right) \triangleleft G$ and is not f.p.f. If $W$ is min. non f.p.f. with respect to $G$ then $W=C\left(W_{0}\right) \cap W$, i.e. $W_{0} \subseteq Z(W)$.

Note that if $C\left(W_{0}\right) \cap W$ is not f.p.f., then it is equal to $W$, since $W$ is a minimal non-f.p.f. group with respect to $G$. In this case we again have $\mathrm{W}_{0} \subseteq \mathrm{Z}(\mathrm{W})$

If $C\left(W_{0}\right) \cap W$ is $f . p . f$. , we must have $C\left(W_{0}\right) \cap W=W_{0}$. Then $C\left(W_{0}\right) \cap W$ is faithful on the minimal $\mathrm{W}_{0}$-spaces, so we can apply (2.7) to conclude that $\mathrm{w}_{0}=\left(\mathrm{W}_{0}\right) \cap \mathrm{W}$ has index $\mathrm{r}$ in $\mathrm{W}$. Suppose that $\mathrm{w}_{0}$ is cyclic of order $\mathrm{w}^{\mathrm{b}}$, 
generated by $v$. We must have $r=b$ and an element $\sigma$ in $w$ such that $\sigma^{-1} \nu \sigma=\nu^{a}, \quad a \neq 1$ and $\nu=\sigma^{-w} \nu \sigma^{w}=\nu^{a^{w}}$, so $a^{w} \equiv 1 \bmod w^{b}$. This implies $\mathrm{a} \equiv 1 \bmod \mathrm{w}^{\mathrm{b}}$ and $\nu^{\mathrm{a}}=1$. That is, $\mathrm{w}$ must centralize $\mathrm{w}_{0}$ and we again have $\mathrm{W}_{0} \subseteq \mathrm{Z}(\mathrm{W})$.

The rest of the proof is essentially the same as for the nonsolvable case. See $(2.3)-(2.6)$ and $(2.8)$.

(3.5) COROLLARY. If the $\mathrm{n}$ is a power of 2 and $|G|$ is odd and $G$ is irreducible then $G$ is cyclic.

PROOF. Put $r=1$ in (3.3) and (3.4). If $r=1$ in conclusion (b) of (3.4) then $G$ is reducible, contrary to hypothesis. (3.6) DEFINITION. Let $S$ be the subgroup of $G$ generated by its 2-elements and let $O(S), O(G)$ the maximal normal subgroups of odd order in $S, G$ respectively.

(3.7) THEOREM. Let $G$ be an irreducible group of linear transformations acting on a vector space of dimension $2^{\mathbf{S}} \mathbf{r}$ over $G F(q)$, where $q$ is a power of 2 and either $r=1$ or $r$ is an odd prime. Then the following hold:

(a) If $O(S)$ is f.p.f., then $O(S)$ is cyclic and the index of $C[O(S)]$ in $G$ divides $2^{S} \mathrm{r}$ unless $\mathrm{C}[0(\mathrm{~S})]$ is not faithful on the minimal $0(\mathrm{~S})$-spaces. (b) If $O(S)$ is not f.p.f. but Fit $O(S)=$ Fit $G$ is f.p.f. then $O(S)$ has a normal cyclic subgroup of index $r$ and the non-f.p.f. elements in $O(S)$ have order $r$.

(c) If Fit $O(S)$ is not f.p.f. then $G$ has a normal subgroup $W$ satisfying the conditions of (3.4).

(d) If $O(S)$ is f.p.f. and $S$ is nontrivial then $S$ contains a subgroup $\mathrm{S}_{1}$ which is generated by its 2-elements and is a minimal (normal) non-f.p.f. subgroup with respect to $G$. If $S_{1}$ is nonsolvable then $s_{1} / 0(s) \cap S_{1}$ is a direct product of isomorphic simple nonabelian groups and $O(S) \cap S_{1}=Z\left(S_{1}\right)$. 
In each of the above $O(S)$ can be replaced by $O(G)$.

PROOF. If $G$ is irreducible then the dimension of a minimal invariant subspace for any normal subgroup must divide the $2^{\mathrm{s}} \mathrm{r}$, so that the previous Lemmas apply to $S$ and $O(S)$ so that (a) - (c) are direct results of the previous Lemmas. Note that a normal subgroup of $G$ does not fix any subspace pointwise if $G$ is irreducible.

In case (d) note that no group of even order is f.p.f. when the characteristic of $F$ is 2 . Hence if $S_{1}$ is a minimal non-f.p.f. group with respect to $G$, then $S_{1}$ must be a minimal nonsolvable normal subgroup of $G$ so that (2.2) applies. The details are left to the reader.

\section{TRANSLATION PLANES.}

(4.1) DEFINITION. In this section $G$ is a subgroup of the linear translation complement of a translation plane $\pi$ of order $q^{d}$, where $q$ is a power of 2 and $d=2^{s-1} r, r$ is an odd prime and $s \geqq 2 . G$ is irreducible and no component of the spread defining $\pi$ has an orbit of length 2 ; no proper subplane has an orbit of length 2 .

REMARK. The dimension of the vector space on which $G$ is defined is $2^{\mathrm{s}} \mathrm{r}=2 \mathrm{~d}$ so that (3.7) applies to G. Translation planes of order $2^{6}$ are frequent possible exceptions to theorems, so the case $q=s=2, r=3$ has special interest.

(4.2) LEMMA. Suppose that $G$ has a normal subgroup $W$, where $W$ is a 3-group. Then $W$ has at least three invariant components; if $\sigma \in W$ is non-f.p.f., then $V(\sigma)$ is a subplane; the partial spread consisting of those elements of the spread fixed by $\mathrm{W}$ is invariant under $\mathrm{G}$.

PROOF. If $\mathrm{q}$ is a power of 2 , then $\mathrm{q}^{2} \equiv 1 \bmod 3$. The number of components of the spread is $q^{d}+1$ so that, if $d$ is even, a 3-group in $G$ must fix at least two components. If $\mathrm{W}$ is normal, $\mathrm{G}$ must permute the components fixed 
by $W$. By (4.1), $W$ has at least three fixed components. The rest of the proof is left to the reader.

(4.3) LEMMA. If $\mathrm{r}=3$ and $\mathrm{G}$ has a normal nonabelian group w-group $\mathrm{W}$ as in (3.4) for some prime $w$ then $w=3$ and $W$ satisfies the conclusions of (4.2). PROOF. See (3.4) and (4.2).

(4.4) LEMMA. If $s=q=2$ in (4.1) and if $G$ has a normal subgroup $W$ where $\mathrm{W}$ is an elementary abelian w-group of order $>\mathrm{w}$, then $w=3$ or 5 and the dimension of a homogeneous $W$-space is 2 or 4 .

PROOF. The vector space on which $\pi$ is defined has dimension $4 \mathrm{r}$, so a minimal $\mathrm{W}$-space has dimension $1,2,4, \mathrm{r}, 2 \mathrm{r}$, or $4 \mathrm{r}$. If $\mathrm{W}$ should.fix a minimal $W$-space pointwise, then $G$ could not be reducible. Thus if a minimal W-space has dimension $i$, then $w$ must divide $2^{i}-1$. Thus $i=1$ is impossible and $w$ divides $2^{4}-1=3.5$ if $i=2$ or 4 .

If a minimal $\mathrm{W}$-space has dimension which is a multiple of $r$ then a homogeneous $\mathrm{W}$-space must also have dimension which is a multiple of $r$. Since $W$ is not faithful on homogeneous W-spaces the case $4 \mathrm{r}$ does not happen (the whole vector has dimension $4 \mathrm{r}$ ).

A subplane in this context is a vector space of even dimension. Thus a homogeneous $\mathrm{W}$-space is either an $\mathrm{r}$-dimensional subspace of a component or has dimension $2 r$. In the latter case there will be just two homogeneous W-spaces, contrary to the assumptions in (4.1) that no component or subplane has an orbit of length two.

We are left with the possibility that there are precisely 4 homogeneous $\mathrm{W}$-spaces and that they are r-dimensional subspaces of four distinct lines. Furthermore, Clifford's theorem implies that every minimal W-space is in one of these 4 homogeneous $W$-spaces. 
Let $\sigma$ be any element of $W$ such that $V(\sigma)$ is nontrivial. Then $V(\sigma)$ must be a Baer subplane. If there are precisely 4 minimal $\mathrm{W}$-spaces then four of the $2^{r}+1$ components of $V(\sigma)$ are invariant under $W$ and $w$ must divide $\left(2^{r}+1\right)-4$. But also we must have $2^{r} \equiv 1 \bmod w$. This implies $w=2$ which is a contradiction. The only possibilities left are $w=3$ or 5 .

We conclude that each homogeneous W-space has dimension 2 or 4 . But W must be faithful and hence $w$ must divide $2^{4}-1=3 \cdot 5$. (4.5) LEMMA. If $\mathrm{d}=6$ in $(4.1)$ and if $G$ has a normal subgroup $W$ where $W$ is an elementary abelian $w$-group of order $>w$ then $|w|=w^{2}$ or $|w|=3^{m}$ with $\mathrm{m} \leqq 4$

PROOF. By (4.4), either $w=3$ or $w=5$ and if $w=5$ there are just 3 homogeneous W-spaces (each of dimension 4 in this case). Let $V_{1}, V_{2}, V_{3}$ be three homogeneous $W$-spaces whose direct sum is invariant under $0(G)$. An element of $W$ which is non-f.p.f. on $V_{1}$ must fix a minimal W-space pointwise and hence fix $V_{1}$ pointwise. An elementary abelian group of order $w^{m}, m>1$ cannot be f.p.f. Hence if $|w|=w^{m}, v_{1}$ is pointwise fixed by a subgroup of order $w^{m-1}$. Suppose $m \geqq 3$. Then the subgroup fixing $V_{1}$ pointwise has a subgroup of order $\mathrm{w}^{\mathrm{m}-2}$ which fixes $\mathrm{V}_{1}$ and $\mathrm{v}_{2}$ pointwise. A similar phenomenon works for each minimal $O(G)$ space; the result is that there is a subgroup of order $w^{m-2}$ whose fixed point space has dimension greater than $d$. (Recall that $\pi$ has order $q^{d}$.) This cannot happen so $w^{m-2}$ must be the identity. If $w=3$ and there are 6 homogeneous $w$-spaces, again take $|W|=w^{m}$. By reasoning to the above there is a subgroup of order $w^{m-4}$ (if $m>4$ ) which fixes four homogeneous $\mathrm{W}$-spaces pointwise. But the pointwise fixed subspace of a nontrivial element cannot have dimension exceeding 6 if $d=6$. Hence $\mathrm{m} \leqq 4$. 
(4.6) LEMMA. Suppose that $r=3$ and $s=2$ and that $G$ has a normal subgroup $\mathrm{W}$ as described in (3.4). Let $\sigma \in \mathrm{W}$ and let $\mathrm{V}(\sigma)$ be nontrivial. Then $\mathrm{V}(\sigma)$ is a subplane.

PROOF. If $W$ is nonabelian apply $(4.3)$. If $W$ is abelian then (4.5) implies that $|W|=w^{2}$. Furthermore, the number of homogeneous $W$-spaces is equal to 3 and each element of $W$ which does not fix a homogeneous W-space pointwise must be f.p.f. on it. The dimension of a homogeneous W-space must divide 4, so $\mathrm{q}^{4}-1 \equiv 0 \bmod w$.

If $\sigma \in \mathrm{W}$ and $\mathrm{V}(\sigma)$ is nontrivial but not a subplane then $\mathrm{V}(\sigma)$ is included in some component $\ell$. Suppose that $V(\sigma)$ has dimension 4 . Then $q^{6}-q^{4}=q^{4}\left(q^{2}-1\right) \equiv 0 \bmod w$ and $q^{2} \equiv 1 \bmod w$. Likewise if $v(\sigma)$. has dimension 1 or $2, q^{2} \equiv 1 \bmod w$.

But if $q^{2} \equiv 1 \bmod w$, then $q^{6}+1 \equiv 2 \bmod w$, so $W$ fixes at least two components of the spread. As in the proof of (4.2), $W$ fixes at least three components so that $V(\sigma)$ must be a subplane.

REMARK. If $\mathrm{q}^{2}+1 \equiv 0 \bmod \mathrm{w}$ we cannot guarantee that any components of the spread are invariant under $w$.

Hering and Ho [5] have investigated the case where $s=2$ and $r$ is odd but not necessarily prime. In their Theorem (5.7), they have given information about S. They come up with the following cases when all involutions are Baer involutions. They also include the situation where there are affine elations in G; the nature of the group generated by affine elations is known from Hering's earlier work [3]. We shall leave this case out, although there is more information than what is implied immediately by Hering's work on elations. They make no assumptions of irreducibility; we state these results in the form that they take if we assume (4.1). 
The Hering and Ho cases (with the restrictions mentioned above):

1. $\mathrm{S} / 0(\mathrm{~S})$ is elementary abelian of order $4 ; \mathrm{C}[0(\mathrm{~S})] \cap \mathrm{S}=\mathrm{z}[0(\mathrm{~S})]$.

2. $\mathrm{S} / 0(\mathrm{~S})=\operatorname{PSL}(2, \mathrm{u}) \mathrm{u} \equiv \pm 3 \bmod 8$.

3. $|\mathrm{s}|$ is not divisible by 4 .

4. ( $\mathrm{S}$ is elementary abelian. This case is void if $G$ is irreducible.)

5. $S \equiv \operatorname{SL}\left(2,2^{b}\right)$ for some b.

We would know more about the action of $G$ on the plane in the first three cases if we knew more about $O(S)$. Here (3.7) of this paper is some help.

These results are sharpened by $(4.3),(4.5)$ and $(4.6)$ if $r=3$ and $s=2$. (The order of the plane is $q^{6}$.) We also have the following:

(4.7) LEMMA. Assume (4.1) and that $\mathrm{r}=3, \mathrm{~s}=2$. Suppose that $\mathrm{S} / 0(\mathrm{~S})=\mathrm{PSL}(2, \mathrm{u})$, where $u \equiv u \pm 3 \bmod 8$. Then $u=3,5,11,13$ or 19 . Furthermore $0(S)$ is trivial unless $u=3$ or 5 .

PROOF. It follows from Harris and Hering [2] that $u \leqq 2 \cdot 12+1=25$. If $u \equiv \pm 3 \bmod 8$, and $u$ is a prime power then $u=3,5,11$, or 13 , or 19 .

Note that $\mathrm{S} / 0(\mathrm{~S})$ is simple unless $\mathrm{u}=3$. Thus if $u \neq 3, \mathrm{~S}$ is a minimal normal nonsolvable subgroup. If $0(S)$ is $f . p . f$. , then $0(S)=Z(S)$ by (3.7)(d). Furthermore $O(S)$ is a subgroup of the Schur multiplier; the Schur multiplier has order 2 so $O(S)$ must be trivial if $u \neq 3$ and $O(S)$ is f.p.f. Suppose that 0 (S) satisfies case (b) of (3.7). Then $0(\mathrm{~S})$ has a normal cyclic subgroup $\mathcal{U}$ of index 3. But $\mathscr{U}$ is normal in $G$; by (2.7) $C$ (थ) has index in $G$ which divides 12. Hence $\mathrm{S} / \mathrm{C}(\mathscr{U}) \cap \mathrm{S}$ is solvable. If $\mathrm{S} / 0(\mathrm{~S})$ is simple this implies that $\mathrm{C}(\mathscr{U}) \cap \mathrm{S}=\mathrm{S}$. This is a contradiction.

Suppose that $O(S)$ satisfies case (c) of (3.7) so that $W$ of (3.4) exists. If $W$ is nonabelian then (4.3) implies that $w=3$ and $W / W_{0}$ is elementary abelian of order 9. Then $S$ induces a subgroup of $\mathrm{SL}(2,3)$ on $\mathrm{W} / \mathrm{W}_{0}$. But $\mathrm{SL}(2,3)$ is solvable, so the centralizer of $\mathrm{W} / \mathrm{W}_{0}$ must be nonsolvable. 
Since $S$ must be a minimal nonsolvable normal subgroup of $G$, this implies that $S$ centralizes $\mathrm{W} / \mathrm{W}_{0}$. The reader may verify that a 2-element centralizes $\mathrm{W} / \mathrm{W}_{0}$ if and only if it centralizes $\mathrm{W}$. But $\mathrm{S}$ is generated by its 2-elements so $\mathrm{S}$ must centralize $\mathrm{W}$. This is a contradiction since $\mathrm{W}$ is nonabelian and in $S$.

If $W$ is elementary abelian then (4.5) and (4.6) apply. Furthermore W has exactly 3 homogeneous subspaces, each of dimension 4 . Let these subspaces be $V_{1}, V_{2}, V_{3}$. The normal subgroup of $G$, call it $H$, which fixes all three must have index dividing 6 , since $G$ induces a transitive permutation group on $\mathrm{V}_{1}, \mathrm{~V}_{2}, \mathrm{~V}_{3}$. Then $\mathrm{S} \cap \mathrm{H}$ has index dividing 6 so $\mathrm{s} / \mathrm{H}$ is solvable. Hence $\mathrm{H} \cap \mathrm{S}$ is a nonsolvable normal subgroup of $G$. Hence $S \subseteq H$. Thus $S$ fixes $V_{1}$ of dimension at most 4. Applying Harris and Hering [2] again, $u \leqq 2 \cdot 4+1=9$. Hence $u=5$.

(4.8) THEOREM. Let $\pi$ be a translation plane of order $q$, where $G F(q)$ is a subfield of the kernel. Let $\sigma$ be an element of the intersection of the translation complement with $\mathrm{GL}(2 \mathrm{~d}, \mathrm{q})$ such that $|\sigma|$ is a prime $u$. Then u divides $q\left(q^{d}+1\right) \prod_{i=0}^{d-1}\left(q^{d-i}-1\right)$. In particular if $q$ is a prime $p$ and $u$ is a prime factor of the translation complement, then $u$ divides $p\left(p^{d}+1\right)^{d-1} \Pi^{d-i}\left(p^{d}-1\right)$ and $u$ cannot be a p-primitive divisor of $p^{e}-1$ for $d<e<2 d$.

PROOF. We may think of $\pi$ as being represented on a vector space of dimension $2 d$ over $G F(q)$. If $u$ does not divıde $q^{2 d}-1$ then $\sigma$ has fixed points other than the zero vector. In particular $\sigma$ fixes some component $l$ of the spread. If $\sigma$ fixes $\ell$ pointwise, then $u$ must divide $q d$. 0 therwise $\ell \cap \mathrm{V}(\sigma)$ is a vector space of some dimension $i$, where $0<i<d$.

Then $\sigma$ must act regularly on the $q^{d}-q^{i}$ points of $l$ not in $V(\sigma)$, so $u$ divides $q^{i}\left(q^{d-i}-1\right)$. But $u$ is prime, so $u$ divides $q\left(q^{d-i}-1\right)$. This establishes the first point of the theorem. The specialization to $q=p$ is 
obvious. It follows from [1] that if $u$ is a prime p-primitive divisor of $\mathrm{p}^{\mathrm{e}}-1$, then $\mathrm{u}$ does not divide $\mathrm{p}^{\mathrm{a}}-1$ for $\mathrm{a}<0<\mathrm{e}$. Furthermore if $\mathrm{u}$ divides $\mathrm{p}^{\mathrm{b}}-1$ then $\mathrm{b}$ is a multiple of $e$ so that $u$ does not divide $\mathrm{p}^{2 \mathrm{~d}}-1$ if $\mathrm{d}<\mathrm{e}<2 \mathrm{~d}$.

(4.9) COROLLARY. If $q=2$ in (4.7), then $u=3,5$, or 13 .

PROOF. The reader may verify that neither 11 nor 19 divides

$$
\left(2^{6}+1\right) \prod_{i=0}^{5}\left(2^{6-i}-1\right) .
$$

\section{REFERENCES}

1. Birkhoff, G. D. and Vandiver, H. S. On the integral divisors of $a^{n}-b^{n}$. Ann. Math. (second series) $\underline{5}$ (1904) 173-180.

2. Harris, M. E. and Hering, C. On the smallest degrees of the projective representations of the groups PSL(n,q) Can. J. Math XXIII, (1971), 90-102.

3. Hering, C. On the shears of translation planes, Abh. Math. Soc. Hamb. 37(1972), 258-268.

4. Hering, C. Zweifach transitive permutationsgruppen, in denen 2 die maximale anzahl von fixpunkten von involutionen ist, Math. $\mathrm{Z}$. $104(1968), 150-174$.

5. Hering, C. and Ho, C. Y. On free involutions in linear groups and collineation groups of translation planes, Fundacão Universidade de Brasilia, Brasilia, Brazil (1977).

6. Huppert, B. Endliche Gruppen I, Springer-Verlag, Berlin and New York (1967).

7. Johnson, N. L. and Ostrom, T. G. Translation planes of characteristic two in which all involutions are Baer, J.Alg. $\underline{54}$ (1978), 291-315.

8. Johnson, N. L. and Ostrom, T. G. The geometry of SL $(2, q)$ in translation planes of even order, Geom. Ded. 8(1979), 39-60.

9. Ostrom, T. G. Finite translation planes, Lecture Notes in Mathematics 158, Springer-Verlag, Berlin and New York, 1970.

10. Ostrom, T. G. Normal subgroups of collineation groups of finite translation planes, Geom. Ded. 2(1974), 467-483.

11. Ostrom, T. G. Collineation groups whose order is prime to the characteristic, Math Z. 156 (1977), 59-71. 
12. Ostrom, T. G. Translation planes of odd order and dimension, Int. J. of Math and Math Sci. 2 (1979), 187-208.

13. Passman, D. S. Permutation groups, W. A. Benjamin, New York, 1968.

14. Schaeffer, H. Translationsebenen, auf denen die Gruppe $\operatorname{SL}\left(2, p^{n}\right)$ operiert. Dimplomarbeit, Universität Tübingen 1975.

15. Walker, M. On translation planes and their collineation groups. Thesis, Westfield College, University of London, 1973. 


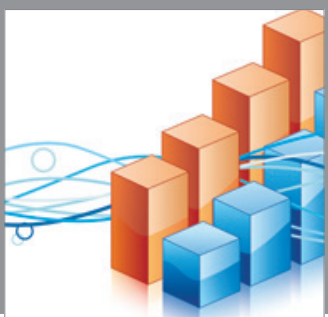

Advances in

Operations Research

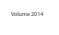

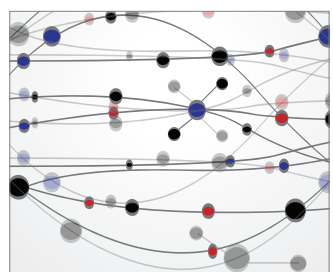

\section{The Scientific} World Journal
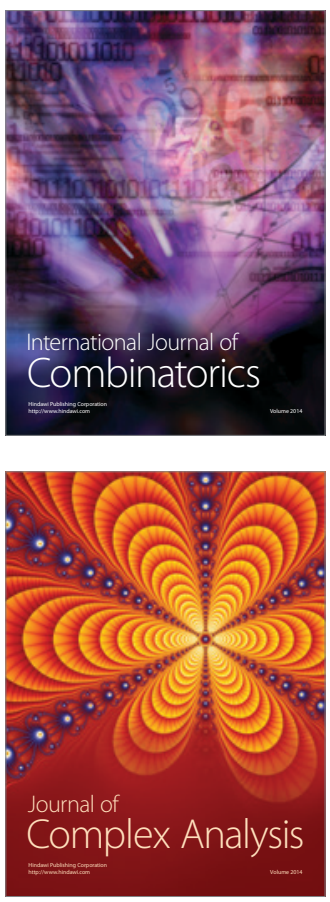

International Journal of

Mathematics and

Mathematical

Sciences


Journal of

Applied Mathematics


Submit your manuscripts at http://www.hindawi.com
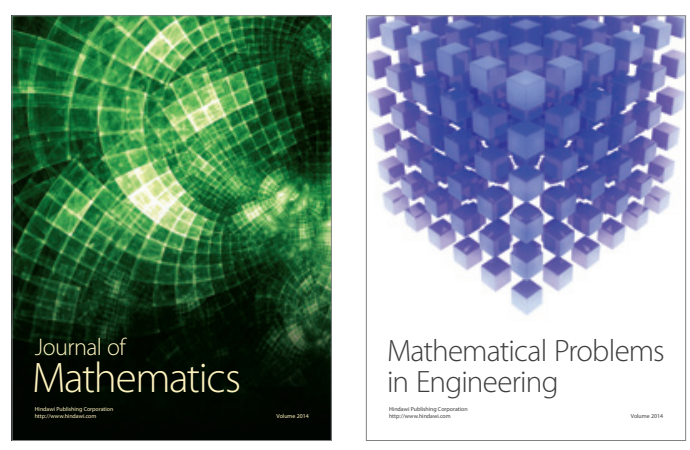

Mathematical Problems in Engineering


Journal of

Function Spaces


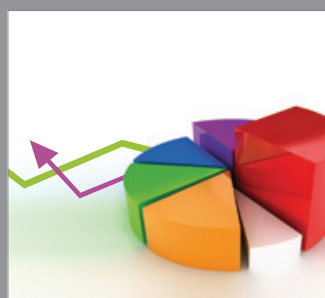

ournal of

Probability and Statistics

Promensencen
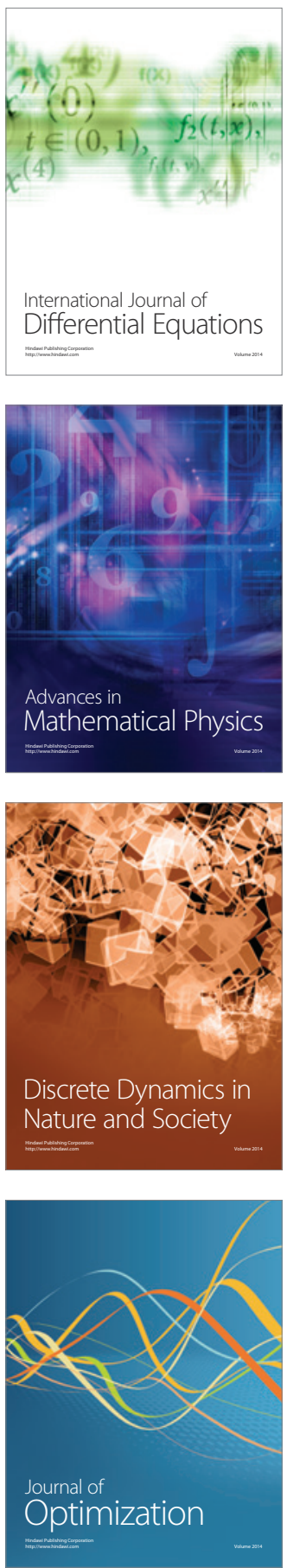